\title{
Analysis of pancreas tissue in a child positive for islet cell antibodies
}

\author{
M. Oikarinen - S. Tauriainen $\cdot$ T. Honkanen $\cdot$ K. Vuori $~$ \\ P. Karhunen • C. Vasama-Nolvi • S. Oikarinen • \\ C. Verbeke • G. E. Blair • I. Rantala • J. Ilonen • \\ O. Simell $•$ M. Knip $\cdot$ H. Hyöty
}

Received: 18 March 2008 / Accepted: 3 July 2008 / Published online: 12 August 2008

(C) Springer-Verlag 2008

\begin{abstract}
Aims/hypothesis Type 1 diabetes is caused by an immunemediated process, reflected by the appearance of autoantibodies against pancreatic islets in the peripheral circulation. Detection of multiple autoantibodies predicts the development of diabetes, while positivity for a single autoantibody is a poor prognostic marker. The present study assesses
\end{abstract}

whether positivity for a single autoantibody correlates with pathological changes in the pancreas.

Methods We studied post mortem pancreatic tissue of a child who repeatedly tested positive for islet cell antibodies (ICA) in serial measurements. Paraffin sections were stained with antibodies specific for insulin, glucagon, somatostatin, interferon alpha, CD3, CD68, cyclooxyge-
M. Oikarinen $(\bowtie) \cdot S$. Tauriainen $\cdot \mathrm{K}$. Vuori $\cdot$ S. Oikarinen $\cdot$

H. Hyöty

Department of Virology, Medical School,

University of Tampere,

Biokatu 10,

FIN-33520 Tampere, Finland

e-mail: maarit.oikarinen@uta.fi

T. Honkanen $\cdot$ I. Rantala

Department of Pathology, Medical School, University of Tampere,

Tampere, Finland

P. Karhunen

Department of Forensic Medicine, Medical School,

University of Tampere,

Tampere, Finland

C. Vasama-Nolvi $\cdot$ I. Rantala

Department of Pathology, Centre of Laboratory Medicine,

Tampere University Hospital,

Tampere, Finland

C. Verbeke

Department of Histopathology,

St James's University Hospital Leeds,

Leeds, UK

\section{G. E. Blair}

Institute of Molecular and Cellular Biology,

Faculty of Biological Sciences, University of Leeds,

Leeds, UK

\author{
J. Ilonen \\ Department of Clinical Microbiology, \\ University of Kuopio, \\ Kuopio, Finland \\ J. Ilonen \\ Immunogenetics Laboratory, University of Turku, \\ Turku, Finland \\ O. Simell \\ Department of Pediatrics, University of Turku, \\ Turku, Finland \\ M. Knip \\ Department of Pediatrics, Tampere University Hospital, \\ Tampere, Finland
}

\author{
M. Knip \\ Hospital for Children and Adolescents, \\ University of Helsinki, \\ Helsinki, Finland \\ H. Hyöty \\ Department of Clinical Microbiology, Centre of Laboratory \\ Medicine, Tampere University Hospital, \\ Tampere, Finland
}


nase-2 (COX-2), beta-2-microglobulin, coxsackie $\mathrm{B}$ and adenovirus receptor (CAR), natural killer and dendritic cells. Apoptosis was detected using Fas-specific antibody and TUNEL assay. Enterovirus was searched for using immunohistochemistry and in situ hybridisation, as well as enterovirus-specific RT-PCR from serum samples.

Results The structure of the pancreas did not differ from normal. The number of beta cells was not reduced and no signs of insulitis were observed. Beta-2-microglobulin and CAR were strongly produced in the islets, but not in the exocrine pancreas. Enterovirus protein was detected selectively in the islets by two enterovirus-specific antibodies, but viral RNA was not found.

Conclusions/interpretation These observations suggest that positivity for ICA alone, even when lasting for more than 1 year, is not associated with inflammatory changes in the islets. However, it is most likely that the pancreatic islets were infected by an enterovirus in this child.

Keywords Islet cell antibodies · Pancreas · Type 1 diabetes

$\begin{array}{ll}\text { Abbreviations } \\ \text { CAR } & \text { coxsackie B and adenovirus receptor } \\ \text { CBV3 } & \text { coxsackievirus B3 } \\ \text { DIPP } & \text { Diabetes Prediction and Prevention study } \\ \text { GADA } & \text { GAD antibody } \\ \text { HSP } & \text { heat shock protein } \\ \text { IA-2A } & \text { islet antigen } 2 \text { antibody } \\ \text { IAA } & \text { insulin autoantibodies } \\ \text { ICA } & \text { islet cell antibodies } \\ \text { JDFU } & \text { Juvenile Diabetes Foundation unit } \\ \text { RU } & \text { relative units }\end{array}$

\section{Introduction}

In the development of type 1 diabetes, the beta cell damaging process seems to progress through different stages. Benign autoimmunity is characterised by the presence of a single autoantibody and/or low titres of autoantibodies, and does not necessarily progress to clinical disease [1]. In contrast, simultaneous detection of several autoantibodies at high titres indicates an aggressive process and predicts progression to overt diabetes. The initiation of the process as well as the shift from benign to aggressive autoimmunity may require environmental influence, which can for example be a virus infection [2] or exposure to certain dietary compounds [3].

One of the greatest gaps in our current knowledge of the disease process in type 1 diabetes is the lack of information about the immunopathological changes affecting the human pancreas during different stages of the process. It is not known how different autoantibody profiles may correlate with the immunological process and the possible presence of viruses in the pancreas. For example, enteroviruses have been linked to autoimmune diabetes in both human and animal studies [2], but our knowledge of the presence of enterovirus in the human pancreas is still very limited. Enteroviruses have been detected in pancreatic islets of patients with type 1 diabetes [4] and in some studies coxsackievirus B4 was isolated from the pancreatic tissue [5-7].

The purpose of this study was to analyse whether early (benign) autoimmunity is associated with changes in islet morphology, markers of the autoimmune process or presence of enterovirus in the pancreas of a child who had repeatedly tested positive for islet cell antibodies (ICA) over a 13 month period.

\section{Methods}

Study material The material in this study comprised sera and pancreatic tissue of a boy taking part in the Diabetes Prediction and Prevention (DIPP) study in Finland. The child was observed from birth, and blood samples were taken at 3- to 6-month intervals. He tested positive for ICA (titres 4-8, Juvenile Diabetes Foundation units [JDFU]), but negative for other diabetes-associated autoantibodies in five sequential serum samples taken over a period of 13 months preceding his accidental drowning and immediate death at the age of 36 months. Pancreatic tissue sample was taken during routine autopsy at Tampere University Hospital (one $1.5 \mathrm{~cm}^{2}$ sample was available from one part of the pancreas). The sample was formalin-fixed, embedded in paraffin and cut in $5 \mu \mathrm{m}$ serial successive sections onto microscopic slides. The child carried HLA-DQB1*02/ *0302 allele conferring increased genetic risk for type 1 diabetes. The study was approved by the National Authority for Medicolegal Affairs in Finland.

In situ hybridisation The presence of enteroviral genome in pancreatic tissue was analysed using in situ hybridisation. An enterovirus-specific oligonucleotide probe (sequence from 5' to 3' GAA ACA CGG ACA CCC AAA GTA GTC GGT TCC GCT GCR GAG TTR CCC RTT ACG ACA) was designed to hybridise with the conserved, groupcommon sequence in the $5^{\prime}$ non-coding region of the enteroviral genome to detect all known enterovirus types. The probe was $3^{\prime}$ end-labelled with digoxigenin using a kit (DIG oligonucleotide tailing kit; Roche Diagnostics, Welwyn Garden City, UK). A 10 pmol sample of the probe was used for one labelling reaction. Hybridisation was performed using earlier published conditions [8]. The amount of probe in the hybridisation cocktail was $250 \mathrm{ng}$, the hybridisation time was $3 \mathrm{~h}$. Binding of the probes was 
revealed by anti-digoxigenin antibody, which was conjugated with alkaline phosphatase. This enzyme together with its substrate, nitro blue tetrazolium/5-bromo-4-chloro-3indolyl phosphate yields an insoluble purple precipitate, which can be detected using a light microscope. Enterovirus-infected and mock-infected green monkey kidney cells were used as controls.

Immunohistochemical staining The following monoclonal antibodies were used for immunohistochemical staining: anti-enterovirus (clone 5-D8/1, 1:1000; DakoCytomation, Glostrup, Denmark), anti-heat shock protein (HSP) 60 (MAB 3514, 1:200; Chemicon International, Temecula, CA, USA), anti-interferon alpha (MMHA-2, 1:50; PBL Biomedical Laboratories, Piscataway, NJ, USA), anti-Fas (MAB4622, 1:15; Chemicon International), anti-CD3 (clone PS1, 1:100; Novocastra Laboratories, Newcastle upon Tyne, UK), anti-CD68 (clone PG-M1, 1:150; DakoCytomation), anti-Ki-67 (clone MIB-1, 1:200; DakoCytomation), antiCD34 (clone NCL-END, 1:500; Novocastra Laboratories), anti-CD56 (clone NCL-CD56-1B6, 1:150; Novocastra Laboratories) and anti-CD23 (clone NCL-CD23-1B12, 1:150; Novocastra Laboratories). The following polyclonal antibodies were used for immunohistochemical stainings: anti-insulin (clone A0564, 1:1500; DakoCytomation), antiglucagon (clone A0565, 1:1500; DakoCytomation), antisomatostatin (A0566, 1:1500; DakoCytomation) and anti-beta-2-microglobulin (ab 15976; 1:1500, Novus Biologicals, Littleton, CO, USA). In-house polyclonal antibodies for echovirus $11(1: 100)$ and coxsackievirus B3 (CBV3) (1:200) were also used for immunohistochemical staining. These antibodies (IgG fraction of rabbit sera) were produced by immunising rabbits with highly purified viruses.

For light microscopic immunoperoxidase staining, $3 \mu \mathrm{m}$ paraffin sections were cut on capillary gap microscope slides (ChemMate; DakoCytomation) or microscope slides (Superfrost Plus; Menzel-Glaser, Brunswick, Germany). Tris-EDTA buffer ( $\mathrm{pH}$ 9.0) retrieval solution was used for insulin, glucagon, somatostatin, HSP60, Fas, CD3, CD68, Ki-67, CD34, CD56 and CD23. Epitope retrieval with $10 \mathrm{mmol} / \mathrm{l}$ citrate buffer ( $\mathrm{pH}$ 6.0) was applied for interferon alpha. Antigen retrieval was performed on re-hydrated sections in a microwave oven at $850 \mathrm{~W}$ for two 7 -min cycles. Beta-2-microglobulin was stained without any pretreatment. Cell conditioning solution (Ventana Medical Systems, Illkirch, France) was used with echovirus 11 and CBV3 stainings for $30 \mathrm{~min}$.

Immunostaining of anti-enterovirus (clone 5-D8/1), HSP60, FAS, CD3, CD68, Ki-67, CD34, CD56, insulin, glucagon, somatostatin and beta-2-microglobulin was performed in an immunostainer (TechMate 500; DakoCytomation) using the labelled avidin-biotin-peroxidase technique or a polymer technique (EnVision; DakoCytomation). Immunostaining with echovirus 11 and CBV3 inhouse antibodies was performed using BenchMark LT (Ventana Medical Systems) and a universal detection system (UltraView; PerkinElmer Life Sciences, Boston, MA, USA). Diaminobenzidine was used as chromogen and haematoxylin as nuclear stain. The specificity of immunohistochemistry was controlled by omitting or replacing the primary antibodies with irrelevant antisera. Known positive tissue samples were also used to confirm the staining reliability of all separate staining batches. Known positive tumour multi-tissue slides were used as positive controls for TUNEL assay and Fas. As a minimum, two parallel sections and about 100 islets were analysed in all immunohistochemical stainings.

Beta cell fractional area The ratio of the beta cell area: exocrine area was measured in the slides stained for insulin using a microscope (Olympus BX61; Olympus America, Melville, NY, USA) and AnalySIS software (Olympus America) as previously described [9].

Confocal microscopy A confocal imaging system (UltraView; PerkinElmer) and laser scanning microscope (LSM 510 Meta; Carl Zeiss Microscopy, Jena, Germany) were used to analyse enterovirus protein levels in insulin-positive and glucagon-positive cells (DakoCytomation). For this purpose, double fluorescence immunostaining was performed by hand on paraffin sections. After antigen retrieval, primary monoclonal mouse anti-enterovirus antibody was localised using Alexa-Fluor 488-conjugated anti-mouse IgG2a (Molecular Probes/Invitrogen, Eugene, OR, USA/ Paisley, UK). Insulin and glucagon were localised by using swine anti-rabbit tetramethylrhodamine isothiocyanate (R0156; DakoCytomation).

Coxsackie B and adenovirus receptor immunostaining Im-

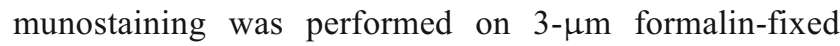
paraffin-embedded tissue sections. Low temperature antigen retrieval was performed as previously described [10] followed by $1 \mathrm{~h}$ incubation with rabbit anti-human coxsackie B and adenovirus receptor (CAR) antibody [11] (diluted 1:1,000). Detection was performed with a reagent (Dako Real Envision K5007; DakoCytomation). Diaminobenzidine was applied as a chromogen (Dako Real DAB plus K5001; DakoCytomation) and Mayer's Haematoxylin for counterstaining. Normal prostatic tissue was used as a positive control, omission of the primary antibody as a negative control.

RT-PCR All follow-up serum samples were analysed using RT-PCR, which detects all known enterovirus serotypes. RNA extraction from serum and stool samples was carried 
out using a kit (QIAmp Viral RNA Mini Kit; Qiagen, Hilden, Germany). The details of the PCR method have been described earlier [12].

Diabetes-associated autoantibodies ICA were analysed by classical immunofluorescence [13]. The detection limit was 2.5 JDFU, and the disease sensitivity $100 \%$ and the specificity $98 \%$ in the most relevant standardisation round [14]. Insulin autoantibodies (IAA), GAD antibodies (GADA) and islet antigen 2 antibodies (IA-2A) were analysed with specific radio-binding assays as described [15]. The cut-off limits were based on the 99th percentile in more than 370 non-diabetic children and corresponded to 3.48 relative units (RU) for IAA, 5.36 RU for GADA and $0.43 \mathrm{RU}$ for IA-2A. The disease sensitivities for the IAA, GADA and IA-2A assays were 58,82 and $72 \%$, respectively, while the corresponding disease specificities were 98\%, 96\% and $100 \%$ in the Diabetes Autoantibody Standardization Program workshop in 2005.

\section{Results}

The structure of the pancreas of the ICA-positive child did not differ from normal in haematoxylin and eosin staining. The islets showed no insulitis and were morphologically intact. Immunohistochemical staining with insulin-specific antibody showed that beta cells were abundant in the islets. The beta cell fractional area relative to exocrine pancreas was $1.8 \%$, which is within the normal range in this age group [16]. Islets also contained cells positive for glucagon and somatostatin. Beta cell replication was not detected in the islets using anti-Ki-67 antibody. Moreover, neither immunohistochemical staining with Fas-specific antibody nor TUNEL assay showed any signs of apoptosis (data not shown).

Immunohistochemical staining with $\mathrm{CD} 3$-specific antibody showed no signs of $\mathrm{T}$ lymphocyte infiltration (Fig. 1a). Macrophages were not detected in the islets using anti-CD68 antibody, while a few macrophages were present in the exocrine pancreas (Fig. 1b). Islets were also negative for cyclooxygenase-2 (Fig. 1c), but strongly produced beta-2-microglobulin, which was also detected in the endothelia of blood vessels (Fig. 1d). Natural killer or dendritic cells were not detected in the islets or exocrine pancreas using anti-CD56 and anti-CD23 antibodies, respectively (Fig. 1e,f).

The pancreatic islets stained clearly positive with the antibody specific for enterovirus VP1 protein (Fig. 2a). Enterovirus positivity was mainly limited to the islets, but some staining was also observed in the endothelia of blood vessels (Fig. 2b,c). Exocrine tissue was mostly enterovirus-
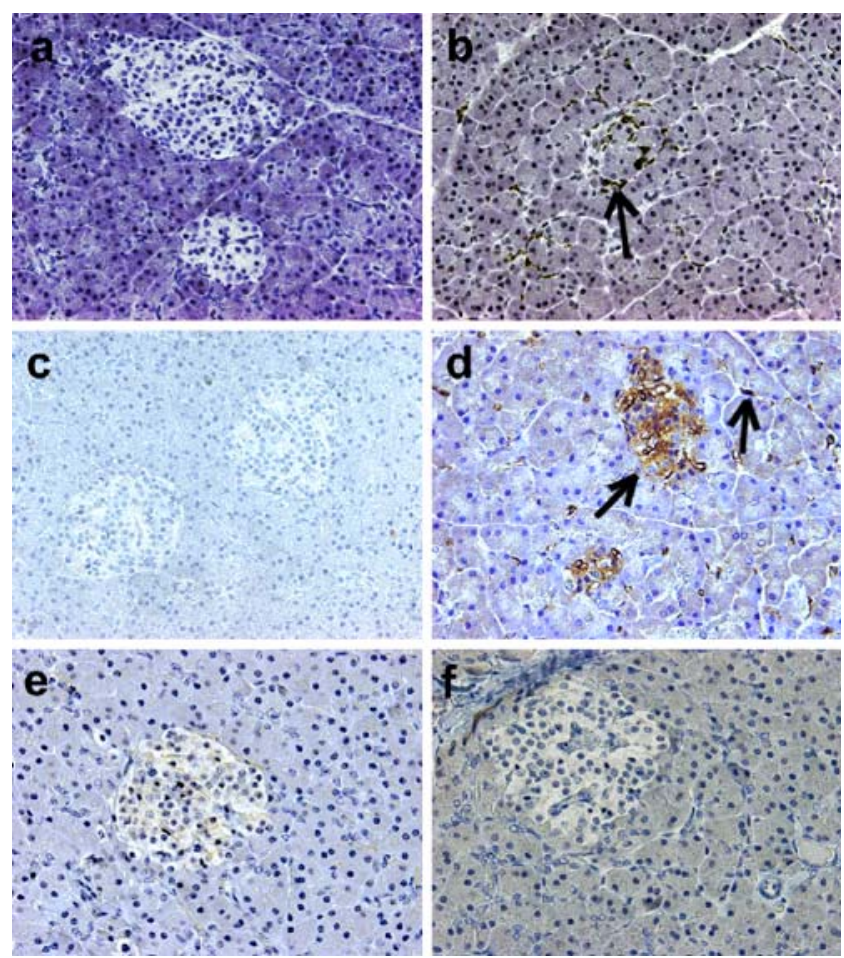

Fig. 1 Immunohistochemical staining with CD3-specific antibody did not show signs of $\mathrm{T}$ lymphocyte infiltration (a). A few cells in the exocrine pancreas stained positive with $\operatorname{CD} 68$ (b) while islets were negative. Staining with cyclooxygenase-2-specific antibody was negative (c), whereas pancreatic islets and the endothelia of blood vessels stained positive with beta-2-microblobulin-specific antibody (d). Staining with natural killer (e) and dendritic cell-specific antibodies (f) was also negative. Some CD68 and beta-2-microglobulin-positive cells are marked with arrows. Magnification $\times 400$

negative. The intensity of islet staining varied and one third of the islets were completely enterovirus-negative. The possible cross-reaction of the enterovirus-specific antibody with HSP60 was excluded by staining the pancreas with the HSP60-specific antibody. HSP60 was strongly produced in exocrine pancreas and in the islets, and staining for HSP60 did not overlap with that obtained by enterovirus-specific antibody (data not shown). The in-house polyclonal echovirus 11-specific antibody followed the same staining pattern: the islets were positive whereas exocrine pancreas remained negative (Fig. 2d). Similarly, the levels of CAR correlated with that of VP1 protein; both were strongly produced in the islets while exocrine pancreas was negative (Fig. 2e). However, staining with CBV3-specific antibody as well as in situ hybridisation were completely negative (data not shown). Staining with IFN-alpha-specific antibody was also negative (Fig. 2f). In addition, all follow-up serum samples of the child remained negative for enterovirus genome as determined by enterovirus-specific RTPCR.

Consecutive slides were stained with antibodies specific for enterovirus, insulin or glucagon to identify enterovirus 


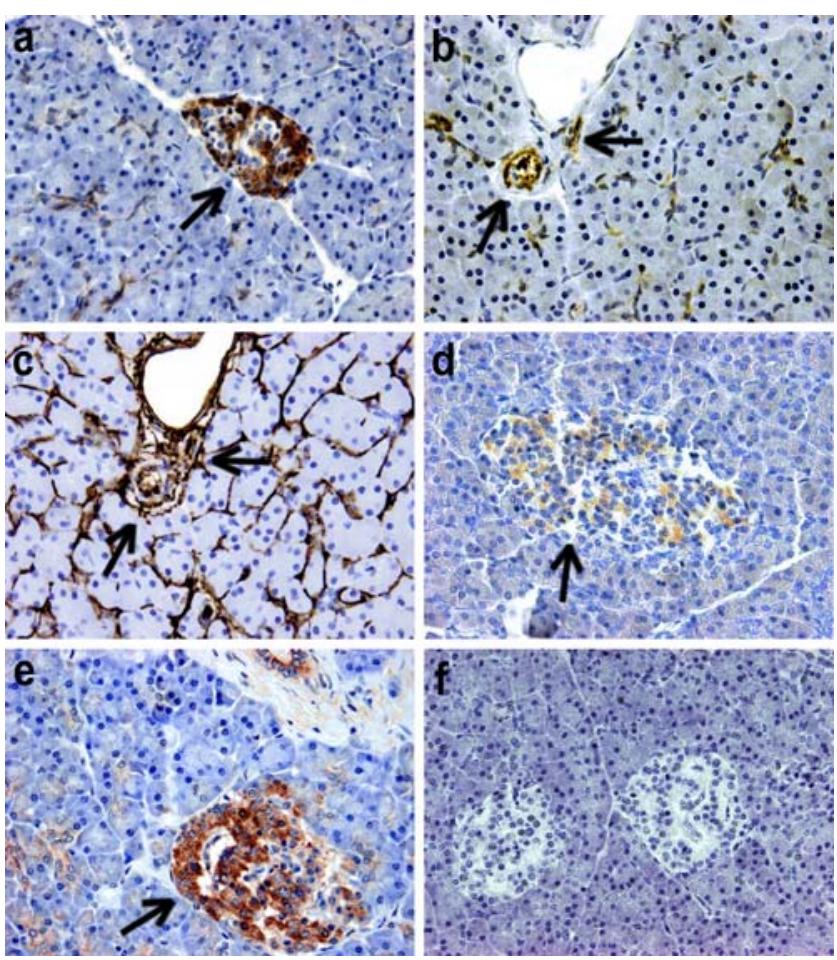

Fig. 2 Enterovirus analysis of the pancreatic tissue of the ICApositive child. Immunohistochemical staining with enterovirus-specific antibody showed positivity in the pancreatic islets (a) and endothelia of blood vessels, as shown by staining the consecutive slides with VP1 (b) and CD34-specific (c) antibody. Islets also stained positive for echovirus 11-specific antibody (d) as well as for CAR (e). Interferon alpha-specific antibody did not show positive staining in islets or exocrine pancreas (f). The arrows indicate a VP1-positive islet (a), blood vessels positive for VP1 (b) and CD34 (c), and islets positive for echovirus 11 (d) and CAR (e). Magnification $\times 400$

localisation in alpha or beta cells and double-staining was used to confirm localisation of the virus. Both methods indicated that enterovirus protein was predominantly produced in glucagon-containing cells, with insulin-containing cells being usually virus-negative (Fig. 3).

\section{Discussion}

We analysed pancreatic tissue of a 3-year old boy positive for ICA. The child participated in the Finnish DIPP study and he tested positive for ICA in five consecutive samples over a 13-month period. Although isolated ICA positivity as such represents a fairly weak indicator of diabetes risk, it may reflect the initiation of the autoimmune process, which in some individuals expands and leads to beta cell damage [1]. At the manifestation of clinical diabetes the pancreatic islets are infiltrated by immune cells [17], but it is not known when this process starts and how it correlates with circulating autoantibodies. The repeated detection of ICA in serial samples, even at relatively low levels, suggests true ICA positivity in this child. Thus, this setting created an excellent opportunity to assess whether such a low-grade humoral beta cell autoimmunity reflects pathogenetic changes in the pancreas.

The results imply that there were no signs of an ongoing autoimmune process in the pancreatic islets in this child. The number of beta cells was not significantly reduced, as the beta cell fractional area relative to exocrine pancreas area was within the normal range for this age group: $1.8 \%$ in this child compared with $2.1 \pm 0.3 \%$ of normal pancreas as described in a recent publication [16]. Beta cell replication was not seen in the islets, which is in contrast to the beta cell replication recently observed in the pancreas of prediabetic adults [18]. Strong islet production of beta-2microglobulin as a part of the HLA class I antigen was the only marker possibly indicating the initiation of a beta celldamaging process. Previous studies have shown that overproduction of HLA class I antigens is one of the earliest markers of the disease process [19]. However, islets were negative for all other inflammation markers tested, such as interferon alpha. Some macrophages were detected in the exocrine pancreas with the CD68-specific antibody, but their number was low and all islets were negative. Accordingly, it seems that, at least in this child, low-grade humoral autoimmunity was not associated with an autoimmune process in the pancreas, supporting the presumption that single autoantibody positivity may reflect harmless nonprogressive beta cell autoimmunity [1]. This is in line with the fact that single autoantibody positivity is a poor prognostic marker for type 1 diabetes. However, we only had one single sample (size $1.5 \mathrm{~cm}^{2}$ ) available from one part of the pancreas. Even though we analysed several parallel sections and about 100 islets for each antibody, we cannot exclude the possibility that a local autoimmune process may have existed in some other part of the organ. This kind of variation in the autoimmune process between different parts of the pancreas has been described previously [20].

The most obvious abnormal finding seen in this child was islet-specific production of enterovirus protein. Immunohistochemical staining with enterovirus-specific antibody was positive in the islets and in the endothelia of some blood vessels, while the exocrine pancreas was negative. This positivity was observed using the same VP1-specific commercial antibody (clone 5-D8/1; DakoCytomation) as that used in previous studies indicating the presence of enterovirus proteins in the islets [21, 22]. Expression of enterovirus proteins in the islets was confirmed using another enterovirus-specific antibody. Although this inhouse antibody was made by immunising rabbits with purified echovirus 11, it also detects other enterovirus serotypes in formalin-fixed cells (S. Tauriainen, unpublished observations). In addition, the VP1-specific clone 5- 


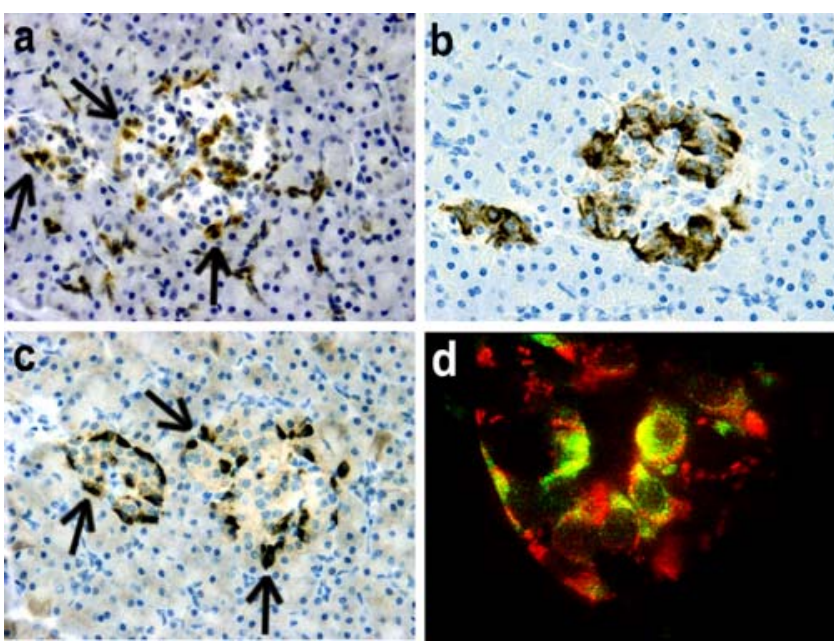

Fig. 3 Immunohistochemical staining of consecutive sections showing the same islet stained with antibodies specific for enterovirus (a), insulin (b) and glucagon (c). Enterovirus protein localised predominantly in glucagon-containing cells while insulin-containing cells were usually negative. Arrows point to the same cells positive for enterovirus (a) and glucagon (c). Co-localisation of enterovirus protein and glucagon was confirmed by double-staining with enterovirus (green) and glucagon-specific (red) antibodies showing as yellow in double-positive cells (d). Insulin-containing cells were negative for the virus in double staining (data not shown). Magnification $(\mathbf{a}-\mathbf{c}) \times 400,(\mathbf{d}) \times 1,000$

D8/1 is known to bind to several enterovirus serotypes [21]. Therefore, on the basis of the current data, we cannot define the precise serotype of the virus infecting the pancreatic islets of this child. The presence of viral protein in endothelial cells is a new finding. Previous studies have shown that enterovirus can cause persistent infection in endothelial cells of blood vessels [23], and it is possible that this is one route through which the virus spreads from the blood to target organs.

The production of enterovirus protein correlated with that of CAR, and indeed both were strongly produced in the islets, but not in the exocrine pancreas. CAR is one of the major enterovirus receptors and mediates the binding and internalisation of coxsackievirus B serotypes. CAR production in islet cells, as observed in this study, confirms a previous report on islet-specific production of this molecule in normal human pancreatic islets [C. Verbeke and G. E. Blair, unpublished results]. This finding fits well with previous observations indicating clear tropism of enterovirus for human islets in patients who died of systemic enterovirus infection or diabetic ketoacidosis; the virus has been repeatedly discovered specifically in the islets, but not in the exocrine pancreas $[4,20,24]$. In addition, enteroviruses infect human islets in vitro. Collectively, these findings support the idea that enteroviruses have tropism to human islets and CAR production may be an important determinant of this tropism. It is also important to note that in the mouse, CAR production is completely the opposite to that seen here, being produced in exocrine pancreas but not in the islets [25]. This probably explains why enteroviruses preferentially infect exocrine pancreas in the mouse, but islets in humans. Further studies are needed to assess whether islet-specific production of CAR could reflect a specific role of coxsackie $\mathrm{B}$ and other CAR-binding enterovirus types in beta cell damage.

The similar staining pattern of CAR and VP1 protein as well as polyclonal echovirus 11-specific antibodies, together with previous findings showing that enteroviruses have strong tropism for pancreatic islets [4], supports the idea that the islets of this child were infected by a coxsackievirus. Enterovirus was predominantly seen in glucagoncontaining alpha cells, while insulin-containing cells were usually negative. This is an interesting finding because alpha cells are not destroyed in type 1 diabetes. One can ask whether beta cells are particularly susceptible to the virus and die once infected, while alpha cells may survive even if they become infected. In a recent study by Dotta et al., similar islet-specific staining was observed in the pancreas of autoantibody-positive participants using the same enterovirus-specific antibody [4]. In that study the presence of virus was confirmed by isolation of coxsackievirus B4 from the islets. In the present study it was not possible to attempt virus isolation because the tissue was treated with formalin, which destroys viral infectivity. We tried to confirm the presence of enterovirus using in situ hybridisation [26]. The negative result in this test may be linked to degradation of viral RNA during post-mortem changes and/or formalin fixation of the tissue. Autopsy was carried out 3 days after death, and it is possible that pancreatic enzymes digested viral RNA while virus proteins remained detectable.

The enterovirus-specific antibody (clone 5-D8/1) has been previously used in studies showing enteroviruses in the pancreas of diabetic patients and in the heart tissue of patients with dilated cardiomyopathy [21, 22, 24]. Even though its specificity has been demonstrated in these studies, it has also been shown that antibodies against enterovirus VP1 protein may cross-react with heat shock proteins, especially HSP60 [27]. We studied possible crossreaction by staining the tissue sample using HSP60-specific antibody. The staining pattern of HSP60 was completely different from the enterovirus staining, as antibodies to HSP60 stained the islets and the exocrine pancreas equally well. This suggests that staining with the enterovirusspecific antibody was not due to cross-reaction with HSP60.

In conclusion, this study shows that single autoantibody positivity is not associated with any local autoimmune process in the pancreas. However, we did find that islet cells selectively produced enterovirus VP1 protein and the major enterovirus receptor, CAR. Further studies are 
needed to define whether certain islet-tropic enterovirus variants are present in the pancreas in individuals who have signs of beta cell autoimmunity.

Acknowledgements We would like to thank E. Tolvanen, A. Karjalainen, E. Eskola and M.-L. Koskinen for technical assistance. J. Booth, St James's University Hospital, Leeds, is thanked for performing the CAR staining. This study was supported by grants from Juvenile Diabetes Research Foundation, Academy of Finland, Päivikki and Sakari Sohlberg's Foundation, Finnish Cultural Foundation, Emil Aaltonen Foundation, The Diabetes Research Foundation and Competitive research funding of the Pirkanmaa Hospital District.

Duality of interest $H$. Hyöty and M. Knip are shareholders in Vactech, which develops vaccines against picornaviruses. All other authors declare that there is no duality of interest associated with this manuscript.

\section{References}

1. Mrena S, Savola K, Kulmala P, Akerblom HK, Knip M, Childhood Diabetes in Finland Study Group (2003) Natural course of preclinical type 1 diabetes in siblings of affected children. Acta Paediatr 92:1403-1410

2. Hyoty H (2002) Enterovirus infections and type 1 diabetes. Ann Med 34:138-147

3. Akerblom HK, Knip M (1998) Putative environmental factors in type 1 diabetes. Diabetes Metab Rev 14:31-67

4. Ylipaasto P, Klingel K, Lindberg AM et al (2004) Enterovirus infection in human pancreatic islet cells, islet tropism in vivo and receptor involvement in cultured islet beta cells. Diabetologia 47:225-239

5. Yoon JW, Austin M, Onodera T, Notkins AL (1979) Isolation of a virus from the pancreas of a child with diabetic ketoacidosis. $\mathrm{N}$ Engl J Med 300:1173-1179

6. Sayama K, Imagawa A, Okita K et al (2005) Pancreatic beta and alpha cells are both decreased in patients with fulminant type 1 diabetes: a morphometrical assessment. Diabetologia 48:15601564

7. Dotta F, Censini S, van Halteren AG et al (2007) Coxsackie B4 virus infection of beta cells and natural killer cell insulitis in recent-onset type 1 diabetic patients. Proc Natl Acad Sci U S A 104:5115-5120

8. Hohenadl C, Klingel K, Mertsching J, Hofschneider PH, Kandolf R (1991) Strand-specific detection of enteroviral RNA in myocardial tissue by in situ hybridization. Mol Cell Probes 5:11-20

9. Butler AE, Janson J, Bonner-Weir S, Ritzel R, Rizza RA, Butler PC (2003) Beta-cell deficit and increased beta-cell apoptosis in humans with type 2 diabetes. Diabetes 52:102-110

10. Reynolds GM, Billingham LJ, Gray LJ et al (2002) Interleukin 6 expression by Hodgkin/Reed-Sternberg cells is associated with the presence of ' $B$ ' symptoms and failure to achieve complete remission in patients with advanced Hodgkin's disease. Br J Haematol 118:195-201

11. McDonald D, Stockwin L, Matzow T, Blair Zajdel ME, Blair GE (1999) Coxsackie and adenovirus receptor (CAR)-dependent and major histocompatibility complex (MHC) class I-independent uptake of recombinant adenoviruses into human tumour cells. Gene Ther 6:1512-1519

12. Lonnrot M, Sjoroos M, Salminen K, Maaronen M, Hyypia T, Hyoty H (1999) Diagnosis of enterovirus and rhinovirus infections by RT-PCR and time-resolved fluorometry with lanthanide chelate labeled probes. J Med Virol 59:378-384

13. Bottazzo GF, Florin-Christensen A, Doniach D (1974) Islet-cell antibodies in diabetes mellitus with autoimmune polyendocrine deficiencies. Lancet 2:1279-1283

14. Lernmark A, Molenaar JL, van Beers WA et al (1991) The Fourth International Serum Exchange Workshop to standardize cytoplasmic islet cell antibodies. The Immunology and Diabetes Workshops and Participating Laboratories. Diabetologia 34:534535

15. Kukko M, Kimpimaki T, Korhonen S et al (2005) Dynamics of diabetes-associated autoantibodies in young children with human leukocyte antigen-conferred risk of type 1 diabetes recruited from the general population. J Clin Endocrinol Metab 90:2712-2717

16. Meier JJ, Butler AE, Saisho Y et al (2008) Beta-cell replication is the primary mechanism subserving the postnatal expansion of beta-cell mass in humans. Diabetes 57:1584-1594

17. Atkinson MA, Maclaren NK (1994) The pathogenesis of insulindependent diabetes mellitus. N Engl J Med 331:1428-1436

18. In't Veld P, Lievens D, De Grijse J et al (2007) Screening for insulitis in adult autoantibody-positive organ donors. Diabetes $56: 2400-2404$

19. Foulis AK (1996) The pathology of the endocrine pancreas in type 1 (insulin-dependent) diabetes mellitus. APMIS 104:161167

20. Foulis AK, McGill M, Farquharson MA, Hilton DA (1997) A search for evidence of viral infection in pancreases of newly diagnosed patients with IDDM. Diabetologia 40:53-61

21. Bourlet T, Gharbi J, Omar S, Aouni M, Pozzetto B (1998) Comparison of a rapid culture method combining an immunoperoxidase test and a group specific anti-VP1 monoclonal antibody with conventional virus isolation techniques for routine detection of enteroviruses in stools. J Med Virol 54:204-209

22. Yousef GE, Brown IN, Mowbray JF (1987) Derivation and biochemical characterization of an enterovirus group-specific monoclonal antibody. Intervirology 28:163-170

23. Conaldi PG, Serra C, Mossa A et al (1997) Persistent infection of human vascular endothelial cells by group B coxsackieviruses. J Infect Dis 175:693-696

24. Foulis AK, Farquharson MA, Cameron SO, McGill M, Schonke H, Kandolf R (1990) A search for the presence of the enteroviral capsid protein VP1 in pancreases of patients with type 1 (insulin-dependent) diabetes and pancreases and hearts of infants who died of coxsackieviral myocarditis. Diabetologia 33:290-298

25. Mena I, Fischer C, Gebhard JR, Perry CM, Harkins S, Whitton JL (2000) Coxsackievirus infection of the pancreas: evaluation of receptor expression, pathogenesis, and immunopathology. Virology 271:276-288

26. Oikarinen M, Tauriainen S, Honkanen T et al (2007) Detection of enteroviruses in the intestine of type 1 diabetic patients. Clin Exp Immunol 151:71-75

27. Harkonen T, Puolakkainen M, Sarvas M, Airaksinen U, Hovi T, Roivainen M (2000) Picornavirus proteins share antigenic determinants with heat shock proteins 60/65. J Med Virol $62: 383-391$ 